\title{
Adequação numérica de nutricionistas e condições higiênico-sanitárias de Unidades de Alimentação e Nutrição Escolares de Maceió, Alagoas
}

\author{
Thaysa Barbosa Cavalcante Brandãoํㅗ Bruna Merten Padilha², Eduarda Larissa Leão de \\ Campos $^{3}$, Girlene Maria de Lima Severiano ${ }^{3}$ e Jacyara Cirilo Leite Torres ${ }^{3}$
}

\begin{abstract}
Objetivou-se avaliar as condições higiênico-sanitárias de Unidades de Alimentação e Nutrição Escolar (UANE) em Maceió e verificar a adequação do número de nutricionistas ao número de alunos matriculados na rede pública de ensino municipal. Trata-se de uma pesquisa transversal e descritiva desenvolvida em 16 dos 32 Centros Municipais de Educação Infantil. A coleta de dados ocorreu entre junho de 2018 e março de 2019. Determinouse o risco sanitário das UANE, a partir da aplicação da "Lista de Verificação para boas práticas na alimentação escolar" elaborada pelo Centro Colaborador em Alimentação e Nutrição Escolar. Verificou-se a adequação do número de profissionais a partir dos parâmetros numéricos mínimos de referência estabelecidos na Resolução CFN no 465/2010. As UANE avaliadas apresentavam inadequações, principalmente no que diz respeito ao uso e a existência de equipamentos para temperatura controlada, sendo a maioria classificada como em risco sanitário regular, de medidas corretivas para a melhoria das condições higiênico-sanitárias das UANE, além da contratação de mais nutricionistas para o monitoramento das atividades na alimentação escolar.
\end{abstract}

Palavras-chave: Alimentação escolar; produção de alimentos; risco sanitário; nutricionistas.

\section{Numerical adequacy of nutritionists and hygienic-sanitary conditions of School Food and Nutrition Units in Maceió, Alagoas}

This work aimed to evaluate the hygienic-sanitary conditions of School Food and Nutrition Units (SFNU) in Maceió and to check the adequacy of the amount of nutritionists to the amount of students enrolled in the public school system. This is a cross-sectional and descriptive research conducted in 16 out of 32 Municipal Centers of Early Childhood Education. Data collection took place between June 2018 and March 2019. The health risk of School Food and Nutrition Units (SFNU) was determined uppon application of a checklist for good practice in school feeding elaborated by the Collaborating Center for School Food and Nutrition. The adequacy of the amount of professionals was verified based on the minimum numerical reference parameters established in Resolution CFN no 465/2010. The evaluated SFNU showed inadequacies, especially with regard to the use and the existence

${ }^{1}$ Doutora em Serviço Social e professora da Universidade Federal de Alagoas no curso de Nutrição (área de Nutrição em alimentação coletiva). Endereço para correspondência: Rua Manoel Maia Nobre no 101, Ed. Portal do Farol, apto. 111, Farol, Maceió - AL. CEP: 57050-120. Tel: (82) 98817351. E-mail: thaysabcb@ hotmail.com. ID ORCID: https://orcid.org/0000-0002-01531044.

2 Doutora em Nutrição. Professora da Faculdade de Nutrição da UFAL, com atuação acadêmica na área de Nutrição em Alimentação Coletiva.

3 Acadêmica de Nutrição pela Universidade Federal de Alagoas. 
of equipment for controlled temperature, most of which were classified as at regular health risk, with percentages of compliance ranging from $44.1 \%$ to $67.9 \%$. The amount of nutritionists (13) was considered inadequate to the amount of students enrolled in the public school system in Maceió. The importance of corrective measures to improve the hygienic-sanitary conditions of SFNU is highlighted, besides the acquisition of more nutritionists for monitoring school feeding activities.

Keywords: School Feeding; food production; health risk; nutritionists.

\section{INTRODUÇÃO}

As Unidades de Alimentação e Nutrição (UAN) são caracterizadas por serem unidades de trabalho ou órgãos de uma empresa que possuem o objetivo de ofertar refeições nutricionalmente equilibradas, microbiologicamente adequadas, isentas de contaminantes e pautadas nos princípios de uma alimentação saudável, visando à promoção, manutenção ou recuperação da saúde de todos os que são beneficiados por seu serviço ${ }^{[1-2]}$.

Para a oferta de uma alimentação segura, é importante a realização do monitoramento do processo produtivo de refeições, a fim de evitar a ocorrência de situações que influenciem diretamente na qualidade sanitária dos alimentos, como contaminação cruzada, higienização incorreta de alimentos e do ambiente, inadequada higiene e conduta pessoal de manipuladores, conservação imprópria de alimentos e utilização de sobras armazenadas incorretamente; as quais promovem a contaminação e a proliferação de microrganismos, comprometendo as condições higiênico-sanitárias das UAN e favorecendo a ocorrência de Doenças Transmitidas por Alimentos (DTA) ${ }^{[3]}$.

Os dados relacionados às DTA são comumente subnotificados em pelo menos $10 \%$. Mesmo assim, no Brasil, entre os anos 2007 e 2018, foram registrados 8.227 surtos de DTA, em que 142.452 pessoas foram afetadas e 136 dos casos resultaram em óbito. No Nordeste, foram observados $1.784(21,68 \%)$ de surtos de DTA, onde 37.042 pessoas foram acometidas, resultando em 37 óbitos, enquanto que, em Alagoas, foram notificados 55 surtos. Dentre os principais locais de ocorrência de surtos, encontram-se as creches e as escolas ${ }^{[4]}$.
As UAN situadas em creches e escolas são denominadas UAN escolares (UANE). Na rede pública de ensino, a oferta de refeições nas UANE é regida pelo Programa Nacional de Alimentação Escolar (PNAE). Esse programa objetiva contribuir para o crescimento e o desenvolvimento biopsicossocial, a aprendizagem, o rendimento escolar e a formação de hábitos saudáveis dos alunos, por meio de ações de educação alimentar e nutricional e da oferta de refeições que cubram as suas necessidades nutricionais durante o período em que permaneçam na escola[5].

O nutricionista é o responsável técnico pelo programa e caberá a ele, dentre outras atribuições, zelar pelas boas práticas higiênicosanitárias no processo produtivo de refeições ${ }^{[6]}$. Contudo, o número de profissionais muitas vezes é insuficiente ao número de alunos e de unidades escolares para monitoramento.

Considerando que o público-alvo do PNAE é potencialmente vulnerável às DTA, a avaliação constante do compromisso das UANE com as medidas de boas práticas higiênico-sanitárias torna-se necessária. Diante do exposto, o presente estudo objetivou avaliar as condições higiênicosanitárias de UANE da cidade de Maceió, Alagoas e verificar a adequação do número de nutricionistas ao número de alunos matriculados na rede pública de ensino municipal.

\section{METODOLOGIA}

Trata-se de uma pesquisa de campo com delineamento transversal e descritivo realizado nas UANE de Centros Municipais de Educação Infantil (CMEI) da cidade de Maceió, Alagoas. Conforme dados do Censo Escolar de 2018, existem 32 CMEI e 37 creches distribuídas em 8 regiões 
administrativas, totalizando 5.709 crianças matriculadas com idade entre 0 e 5 anos. Foram selecionados, aleatoriamente, $50 \%$ das CMEI, totalizando 16 amostras. A coleta de dados ocorreu entre junho de 2018 e março de 2019.

Para avaliar as condições higiênicosanitárias das UANE, foi utilizado o instrumento "Lista de Verificação para boas práticas na alimentação escolar" elaborado pelo Centro Colaborador em Alimentação e Nutrição Escolar (CECANE/UFRGS/UNIFESP) e validado por Stedefeldt et al.[7]. Essa lista é composta por 99 itens divididos em 6 blocos temáticos: edifícios e instalações da área de preparo de alimentos; equipamentos para temperatura controlada; manipuladores; recebimento; processo e produções; e higienização ambiental.

A equipe responsável pela aplicação da lista em cada UANE foi treinada previamente. A partir da tabulação dos dados no software do CECANE/FNDE[8], foi gerado o diagnóstico das condições higiênico-sanitárias de cada UANE, considerando o risco sanitário global e por bloco, o qual foi categorizado, segundo a pontuação $(\%)$ obtida, em: muito alto (0 a $25 \%$ ); alto (26 a $50 \%$ ); regular (51 a 75\%); baixo (76 a 90\%) ou muito baixo (91 a $100 \%)^{[7]}$.

Para verificar o percentual de adequação do número de nutricionistas ao número de alunos matriculados na rede pública de ensino municipal levaram-se em consideração os parâmetros numéricos mínimos de referência estabelecidos pela Resolução CFN no 465/2010[6].

A presente pesquisa foi realizada com a anuência da Secretaria Municipal de Educação de Maceió. A identificação de cada CMEI participante foi preservada por meio de codificação alfabética.

\section{RESULTADOS}

De acordo com os resultados da Tabela 1 , verifica-se que a maioria das UANE avaliadas se encontrou em situação de risco sanitário regular, tendo a UANE A recebido a pontuação mais alta (67,9\%). As UANE N e P foram as classificadas em situação de alto risco $(44,2 \%$ e $44,1 \%$, respectivamente). O bloco que recebeu melhor avaliação em todas as UANE foi o de recebimento, enquadrando-as na classificação de risco sanitário muito baixo. O bloco de pior avaliação, por outro lado, foi o de equipamentos para temperatura controlada, com as UANE enquadradas entre risco sanitário muito alto a regular.

No bloco de edifícios e instalações da área de preparo de alimentos, a maioria $(\mathrm{n}=11,68,75 \%)$ apresentou risco sanitário regular. Entre as inadequações identificadas pode-se citar a ausência de ralos dotados de mecanismos de fechamento automático; ausência de proteção inferior nas portas; paredes e tetos que não apresentam acabamento liso, impermeável, lavável, de cor clara e com presença de sujidades, umidade, bolor e descascamento.

No bloco de equipamentos para temperatura controlada, $68,75 \%(\mathrm{n}=11)$ das UANE se encontraram entre risco sanitário alto e muito alto. As inadequações para este bloco se deram principalmente devido à ausência de termômetro de aferição de temperatura de alimentos, o que impossibilita o controle de temperatura tanto no armazenamento quanto durante a produção. Outro problema foi a ausência de geladeiras ou câmaras frias em número suficiente. 
Tabela 1. Adequação (\%) das condições higiênico-sanitárias, geral e por bloco avaliado, de Unidades de Alimentação e Nutrição Escolares (UANE) de Maceió, Alagoas, 2019

\begin{tabular}{|c|c|c|c|c|c|c|c|}
\hline \multirow[b]{2}{*}{ UANE } & \multicolumn{7}{|c|}{ Bloco avaliado* } \\
\hline & 1 & 2 & 3 & 4 & 5 & 6 & $\begin{array}{c}\text { geral } \\
(\%)\end{array}$ \\
\hline $\mathrm{A}$ & 74,7 & 13,3 & 83,3 & 100,0 & 63,8 & 84,2 & 67,9 \\
\hline B & 78,8 & 46,6 & 76,9 & 100,0 & 40,5 & 64,3 & 62,7 \\
\hline $\mathrm{C}$ & 50,6 & 55,5 & 76,9 & 100,0 & 48,3 & 43,9 & 61,5 \\
\hline $\mathrm{D}$ & 57,0 & 20,0 & 46,1 & 100,0 & 77,7 & 54,8 & 59,0 \\
\hline $\mathrm{E}$ & 70,1 & 33,3 & 58,3 & 100,0 & 44,2 & 31,6 & 53,0 \\
\hline $\mathrm{F}$ & 71,3 & 69,2 & 75,0 & 100,0 & 48,5 & 51,2 & 65,9 \\
\hline G & 58,6 & 26,7 & 53,8 & 100,0 & 49,0 & 81,6 & 56,2 \\
\hline $\mathrm{H}$ & 37,3 & 22,2 & 53,8 & 100,0 & 51,7 & 50,0 & 51,0 \\
\hline I & 48,1 & 61,5 & 46,1 & 100,0 & 57,6 & 64,3 & 60.6 \\
\hline $\mathrm{J}$ & 68,4 & 46,7 & 84,6 & 75 & 31,8 & 77,8 & 60,9 \\
\hline $\mathrm{K}$ & 38,2 & 30,7 & 58,3 & 100 & 53,1 & 94,7 & 58,4 \\
\hline $\mathrm{L}$ & 59,1 & 13,3 & 83,3 & 100 & 65,3 & 84,2 & 66,7 \\
\hline M & 52,3 & 40 & 50 & 100 & 50,3 & 52,4 & 54,1 \\
\hline $\mathrm{N}$ & 57,5 & 22,5 & 50 & 100 & 38,4 & 50 & 44,2 \\
\hline $\mathrm{O}$ & 63,2 & 11,8 & 38.5 & 75 & 39,3 & 64,9 & 44,1 \\
\hline $\mathrm{P}$ & 67 & 23,5 & 66,7 & 100 & 62,9 & 60,5 & 61,8 \\
\hline
\end{tabular}

Para o bloco de manipuladores, grande parte das UANE ( $\mathrm{n}=10,62,5 \%)$ encontrou-se entre risco sanitário alto e regular. As principais inadequações identificadas foram ausência de uniforme completo; presença de adornos; e ausência de renovação de exames médicos periodicamente.

No tocante ao bloco de recebimento, a maioria das UANE se encontrava em risco sanitário muito baixo. As únicas UANE que apresentaram inadequação foram as UANE $\mathrm{J}$ e $\mathrm{O}$. As não conformidades encontradas foram: não efetuar a devolução de produtos reprovados no ato do recebimento e não fazer a segregação e identificação dos mesmos para providências posteriores; e não avaliar a qualidade dos alimentos no ato da entrega.

No bloco de processos e produções, a maioria $(\mathrm{n}=9 ; 56,2 \%)$ das UANE avaliadas foram classificadas em risco sanitário alto. Entre as inadequações estavam a ausência de etiquetas de acordo com a rotulagem original e prazo de utilização; presença de produtos com validade vencida; ausência de armazenamento de amostras; processo de descongelamento inadequado; e a ausência do manual de boas práticas e dos procedimentos operacionais padronizados obrigatórios para serviços de alimentação.

No bloco de higienização ambiental, $68,75 \%(\mathrm{n}=11)$ das UANE se encontraram entre risco sanitário regular e alto. Entre as inadequações para este item, foram identificadas: ausência de documentação que comprove o controle integrado de pragas e vetores; ausência de higienização das esponjas de louça; desinfecção química inadequada de utensílios e equipamentos; ausência de manutenção da higiene ambiental por meio de adequadas e aprovadas técnicas de limpeza, enxágue e desinfecção; presença da prática de varrer o piso a seco na área de produção; e a utilização de panos para secagem de utensílios e equipamentos.

Identificou-se que Maceió conta com 1 nutricionista responsável técnico e 12 nutricionistas no quadro técnico, com atuação de pelo menos 30 horas semanais. Levando-se em consideração apenas o número de alunos matriculados em CMEI e creches, esse número de profissionais estaria com $118,2 \% \%$ de adequação em relação aos parâmetros numéricos mínimos de referência estabelecidos na Resolução CFN no 465/2010 (Tabela 2). 
Tabela 2. Adequação (\%) do número de nutricionistas da rede pública de ensino de Maceió, Alagoas, aos parâmetros numéricos mínimos de referência estabelecidos na Resolução CFN no 465/2010

\begin{tabular}{ccccc}
\hline \multirow{2}{*}{ Modalidade de ensino } & $\begin{array}{c}\text { No de alunos } \\
\text { matriculados* }\end{array}$ & \multicolumn{2}{c}{ № de nutricionistas } & \multirow{2}{*}{$\begin{array}{c}\text { Adequação } \\
(\%)\end{array}$} \\
\cline { 3 - 4 } & 5.709 & Na rede & Ideal** & 118,2 \\
Creche e pré-escola & 53.287 & $1 \mathrm{RT}+12 \mathrm{QT}$ & $1 \mathrm{RT}+10 \mathrm{QT}$ & 56,5 \\
\hline Todas & $1 \mathrm{RT}+12 \mathrm{QT}$ & $1 \mathrm{RT}+22 \mathrm{QT}$ & \\
\hline
\end{tabular}

№ = número; RT = Nutricionista Responsável Técnico; QT = Nutricionistas do quadro técnico

*Segundo o censo escolar de 2018.

**De acordo com os parâmetros numéricos mínimos de referência estabelecidos na Resolução CFN 465/2010[6].

Contudo, sabendo-se que o município assiste a mais de 50 mil alunos, verifica-se a inadequação de $56,5 \%$ do quadro técnico em relação aos parâmetros numéricos mínimos de referência estabelecidos na Resolução CFN no $465 / 2010$ (Tabela 2).

\section{DISCUSSÃO}

A lista de verificação aplicada nas UANE considera como parâmetro de conformidade os procedimentos descritos na RDC no 216/2004[9]. Esta legislação estabelece os procedimentos de Boas Práticas para serviços de alimentação a fim de garantir as condições higiênico-sanitárias durante todo o processo produtivo e distribuição do alimento preparado.

$\mathrm{O}$ presente estudo evidenciou que as UANE dos CMEI da cidade de Maceió se encontram em situação de risco sanitário regular, demonstrando a necessidade de regularização das condições higiênico-sanitárias desses locais. A avaliação por blocos permitiu identificar as não conformidades de maior criticidade e que precisam, portanto, de intervenção imediata.

O bloco de recebimento de todas as UANE foi o que apresentou menor comprometimento sanitário. Soares et al.[10], ao analisarem as condições higiênico-sanitárias de nove UANE de um município do Rio de Janeiro, identificaram que a maioria $(n=8)$ das UANE avaliadas estavam adequadas quanto ao recebimento. Contudo, os autores destacaram como limitação do estudo o fato de que não houve o acompanhamento das atividades relacionadas a esta etapa e sim obtenção indireta dos dados, por meio de indagação às merendeiras sobre os procedimentos adotados, o que pode ter influenciado nesse resultado. Tal limitação também se aplica a esse estudo, de modo que resultados diferentes para esse bloco poderiam ter sido encontrados, caso os dados relativos ao recebimento tivessem sido obtidos por observação direta. Oliveira et al.[11], por sua vez, acompanharam o recebimento de gêneros nas UANE avaliadas e destacaram a ausência de verificação de validade e integridade das embalagens dos gêneros alimentícios como problemas comuns durante o recebimento.

Mezzari \& Ribeiro[12], em um estudo que avaliou as condições microbiológicas em uma escola municipal do Município de Campo Mourão (PR), relataram que investimentos em estrutura adequada e adoção de medidas de higienização podem resultar em maior segurança microbiológica nos estabelecimentos de alimentação coletiva. Salientase que os resultados do presente estudo apontam para falhas nos procedimentos de higienização.

No que se referem às temperaturas controladas dos alimentos e equipamentos, os resultados apontam para inadequações relacionadas principalmente à falta de monitoramento das temperaturas nas etapas de armazenamento, produção e distribuição, por indisponibilidade de termômetro compatível para essa atividade. De acordo com a RDC no 216/ 2004[9] a temperatura das matérias-primas, ingredientes e equipamentos devem ser monitoradas e registradas durante as etapas de recepção e armazenamento. Ademais, as temperaturas dos alimentos prontos para consumo 
devem ser monitoradas e mantidas em valores $\geq 60^{\circ} \mathrm{C}$ para alimentos quentes e $\leq 5^{\circ} \mathrm{C}$ para alimentos frios ${ }^{[9,13]}$. Destarte, a aplicação de medidas de controle rigoroso da temperatura, promovendo a segurança dos alimentos durante estas etapas é uma forma de minimizar o risco de ocorrência de DTA.

Almeida et al.[14] evidenciaram inadequações quanto à avaliação dos manipuladores de escolas e creches, destacando que os itens mais frequentes foram o uso incorreto do uniforme, ausência da touca de proteção para cabelos e de realização de exames médicos periódicos, corroborando com os resultados do presente estudo.

Entre as inadequações encontradas no bloco de higienização ambiental estão a desinfecção química inadequada de utensílios e equipamentos e a utilização de panos para secagem de utensílios e equipamentos, também evidenciadas por Oliveira et al.[11], que relataram que os utensílios não eram submetidos aos processos adequados de lavagem e higienização. Mendes et al.[15] avaliaram a contaminação por Bacillus cereus de 24 utensílios e dos 6 equipamentos mais utilizados em um restaurante de uma universidade pública de Minas Gerais, utilizando o método da esponja, a partir do umedecimento das mesmas em água peptonada a $0,1 \%(\mathrm{p} / \mathrm{v})$ e de sua fricção sobre as superfícies. A presença desse microrganismo patogênico foi detectada em 38,3\% das amostras, indicando a necessidade de medidas mais rigorosas para o seu controle.

Os manipuladores são a principal via de contaminação dos alimentos, o que pode levar ao surto de DTA. Dessa forma, a capacitação desses profissionais quanto às boas práticas de manipulação de alimentos deve ser contínua para que os mesmos adquiram hábitos higiênicosanitários adequados e os apliquem no dia a dia[16].

De acordo com a legislação sanitária ${ }^{[9]}$ os serviços de alimentação devem dispor de Manual de Boas Práticas e de Procedimentos Operacionais Padronizados (POP). No entanto, nenhuma das UANE tinha estes documentos. O Manual de Boas Práticas é um documento que descreve a situação real das operações e dos procedimentos realizados pelo estabelecimento e o POP descreve de forma objetiva as instruções sequenciais para a realização de operações rotineiras e específicas na manipulação de alimentos ${ }^{[0]}$. O propósito da elaboração e implementação destes documentos é estabelecer uma sistemática para garantir a segurança do alimento preparado, portanto são de extrema relevância para orientação dos manipuladores de alimentos e devem estar acessíveis a eles.

A adequação do número de nutricionistas ao número de alunos matriculados na rede pública de ensino municipal também se faz necessária, visto que, conforme a Resolução do CFN 465/2010[6], para atender a modalidade de Educação Infantil, a rede escolar deve ter um nutricionista para cada 500 alunos e que não há, no setor de Alimentação Escolar, profissionais exclusivos para atuação nessa modalidade de ensino.

Os resultados apresentados nesta pesquisa corrobora com Correia et al.[17], que ao verificarem a adequação dos parâmetros numéricos mínimos de referência estabelecidos pela Resolução CFN no $465 / 2010^{[6]}$ às atribuições obrigatórias do nutricionista da região Sul do Brasil, identificaram inadequação do quadro técnico de nutricionistas em $71,6 \%(\mathrm{n}=83)$ dos municípios.

Com base nas inadequações encontradas no diagnóstico, foi elaborado um plano de ação corretiva para cada UANE. Esse documento foi apresentado aos gestores escolares, manipuladores de alimentos e nutricionistas dos CMEI inseridos na pesquisa, em um momento de sensibilização sobre a importância da adoção de boas práticas na manipulação de alimentos. Por fim, os manipuladores de alimentos participaram de uma oficina com carga horária de 4 horas, com atividades práticas e exposição teórica sobre os procedimentos e as etapas de produção que envolvem as boas práticas na manipulação de alimentos.

Frente ao exposto, almeja-se que estas ações nas UANE possam contribuir para o cumprimento dos requisitos estabelecidos pela legislação a fim de garantir as condições higiênicosanitárias do alimento preparado e servido aos escolares, prevenir a ocorrência de DTA e, consequentemente, favorecer a promoção da saúde no âmbito da alimentação coletiva. 
Salienta-se que é responsabilidade do nutricionista acompanhar, fiscalizar e implantar as boas práticas de higiene durante todo o processo produtivo, sendo imprescindível para isso que o número de profissionais esteja adequado. Além disto, o número inadequado de profissionais pode acarretar na sobrecarga de trabalho e comprometer outras atribuições previstas.

\section{CONCLUSÃO}

Verificou-se que a maioria das UANE foi classificada com risco sanitário regular, ou seja, cumpriram apenas parcialmente as exigências legais. A avaliação por bloco identificou que existem alguns pontos que demandam maior atenção, ressalta-se os equipamentos para temperatura controlada em que a maioria das UANE apresentou resultados enquadrados em risco sanitário alto e muito alto $\mathrm{O}$ que pode interferir na segurança desejada para a qualidade da alimentação escolar.

O número de nutricionistas em relação ao número de alunos matriculados nas CMEI é adequado. Contudo, esses profissionais atendem também aos alunos das demais modalidades de ensino. Assim se torna necessário rever o quadro adequando-o ao número total de alunos matriculados na rede pública de ensino.

Além disso, é preciso que medidas corretivas sejam tomadas para a melhoria das condições higiênico-sanitárias das UANE, dentre as quais: investimento em estrutura adequada; adoção de medidas de higienização eficientes para garantir a segurança microbiológica; controle rigoroso de temperatura ao longo de todo o processo de produção das refeições; adoção de técnicas corretas de manipulação dos alimentos; e a conscientização dos profissionais envolvidos no processo de produção do alimento. Tais medidas são fundamentais para a garantia de uma alimentação segura para os comensais.

\section{REFERÊNCIAS}

[1] São José JFB, Coelho AIM, Ferreira KR. Avaliação das boas práticas em unidade de alimentação e nutrição no município de Contagem-MG. Alim. Nutr. [Internet]. 2011 [acesso em 2020 mar 02]; 22(3):479-487. Disponível em: https://www.locus.ufv.br/bitstream/handle/123456789/1749 3.

[2] Fonseca KZ, Santana GR. Guia prático para gerenciamento de unidades de alimentação e nutrição [Internet]. Bahia: Editora UFRB; 2012 [acesso em 2020 mar 02]. Disponível em: http://www.repositorio.ufrb.edu.br/bitstream/123456789/80 4/1/guia $\% 20$ pratico $\% 20$ de $\% 20$ unidade $\% 20$ de $\% 20$ alimantaca o $\% 20 \mathrm{e} \% 20$ nutricao $\% 281 \% 29$.pdf.

[3] Santos IGP, Bezerra VM. Segurança de alimentos em cozinhas escolares do Munícipio de Vitoria da Conquista, Bahia. Revista Baiana de Saúde Pública [Internet]. 2015 [acesso em 2020 mar 02]; 39(2):205-224. Disponível em: http://rbsp.sesab.ba.gov.br/index.php/rbsp/article/download $/ 1527 / 1232$.

[4] Brasil. Ministério da Saúde. Doenças Transmitidas por Alimentos. Situação epidemiológica - doenças transmitidas por alimentos: Banco de dados 2000 a 2018 [Internet]. 2020 [acesso em 2020 mar 31]. Disponível em: https://saude.gov.br/saudede-a-z/doencas-transmitidas-por-alimentos.

[5] Brasil. Ministério da Educação. Fundo Nacional de Desenvolvimento da Educação (FNDE). Cartilha Nacional de Alimentação Escolar [Internet]. Brasília: ME; 2015 [acesso em 2020 mar 02]. Disponível em: https://www.fnde .gov.br/index.php/programas/pnae/pnae-area-gestores/pnaemanuais-cartilhas/item/6820-cartilha-pnae-2015.

[6] Conselho Federal de Nutricionistas (CFN). Resolução CFN no 465/2010. Dispõe sobre as atribuições do nutricionista, estabelece parâmetros numéricos mínimos de referência no âmbito do Programa de Alimentação Escolar e dá outras providências. Diário Oficial da União; 2010 ago 25.

[7] Stedefeldt E, Cunha DT, Silva Júnior EA, Silva SM, Oliveira ABA. Instrumento de avaliação das Boas Práticas em Unidades de Alimentação e Nutrição Escolar: da concepção à validação. Ciênc. Saúde Coletiva [Internet]. 2013 [acesso em 2020 mar 02]; 18(4):947-953. Disponível em: https://doi.org/10.1590/S141381232013000400006

[8] CECANE/FNDE. Ferramentas para as Boas Práticas na Alimentação Escolar, versão 1.0; 2012.

[9] Brasil. Agência Nacional de Vigilância Sanitária. Resolução RDC no 216/2004. Dispõe sobre Regulamento Técnico de Boas Práticas para Serviços de Alimentação. Diário Oficial da União; 2004 set 15.

[10] Soares DSB, Henriques P, Ferreira DM, Dias PC, Pereira S, Barbosa RMS. Boas Práticas em Unidades de Alimentação e Nutrição Escolares de um município do estado do Rio de Janeiro - Brasil. Ciênc. Saúde Coletiva [Internet]. 2018 [acesso em 2020 mar 02]; 23(12):4077-4083. Disponível em: https://doi.org/10.1590/1413-812320182312.23992016. 
[11] Oliveira ABA, Cunha DT, Stedefeldt E, Capalonga R, Tondo EC, Cardoso MRI. Hygiene and good practices in school meal services: Organic matter on surfaces, microorganisms and health risks. Food Control [Internet]. 2014 [acesso em 2020 mar 02]; 40:120-126. Disponível em: https://doi.org/10.1016/j.foodcont.2013.11.036.

[12] Mezzari MF, Ribeiro A. Avaliação das condições higiênicosanitárias da cozinha de uma escola municipal de Campo Mourão - Paraná. SaBios-Revista de Saúde e Biologia [Internet]. 2012 [acesso em 2020 mar 09]; 7(3):60-66. Disponível em: http://revista2.grupointegrado.br/revista/index.php/sabios2/ article/view/647/460.

[13] Monteiro MAM, Ribeiro RC, Fernandes BDA, Sousa JFR, Santos LM. Controle das temperaturas de armazenamento e de distribuição de alimentos em restaurantes comerciais de uma instituição pública de ensino. DEMETRA: Alimentação, Nutrição \& Saúde [Internet]. 2014 [acesso em 2020 mar 02]; 9(1):99-106. Disponível em: https://doi.org/10.12957/demetra.2014.6800.

[14] Almeida KM, André MCP, Campos MRH, Díaz MEP. Condições físico-funcionais e higiênico-sanitárias das unidades de alimentação e nutrição de escolas da Região Centro-Oeste, Brasil. Rev. Nutr. [Internet]. 2014 [acesso em 2020 mar 02]; 3(27):343-356. Disponível em: https://doi.org/10.1590/141552732014000300008 .

[15] Mendes RA, Coelho AIM, Azeredo RMC. Contamination by Bacillus cereus on equipment and utensil surfaces in a food and nutrition service unit. Ciência \& Saude Coletiva [Internet]. 2011 [acesso em 2020 mar 02]; 16(9):3933-3938. Disponível em: https://doi.org/10.1590/S1413-81232011001000030.

[16] Mello AG, Gama MP, Marin VA, Colares LGT. Conhecimento dos Manipuladores de Alimentos sobre Boas Práticas nos Restaurantes Públicos Populares do Estado do Rio de Janeiro. Braz. J. Food Technol [Internet]. 2010 [acesso em 2020 mar 02]; 13(1):60-68. Disponível em: https://www.arca.fiocruz.br/handle/icict/12076.

[17] Corrêa R da S, Rockett FC, Rocha PB, Silva VL, Oliveira VR. Atuação do nutricionista no Programa Nacional de Alimentação Escolar na região Sul do Brasil. Ciênc. Saúde Coletiva [nternet]. 2017 [acesso em 2020 mar 02]; 22(2):563_ 574. Disponível

em: https://www.scielo.br/pdf/csc/v22n2/1413-8123-csc-22-020563.pdf. 